\title{
A Distinct Phenotype of Leg Hyperreflexia in a Japanese Family with Gerstmann-Sträussler-Scheinker Syndrome (P102L)
}

Takanori Takazawa, Ken Ikeda, Hirono Ito, Joe Aoyagi, Yoshikazu Nakamura, Ken Miura, Konosuke Iwamoto, Osamu Kano, Kiyokazu Kawabe and Yasuo Iwasaki

\begin{abstract}
Gerstmann-Sträussler-Scheinker Syndrome (GSS) is an inherited prion disease characterized by midlife onset and slowly progression of cerebellar ataxia and dementia. We report a distinct phenotype of leg hyperreflexia in a Japanese family with GSS. A 38-year-old woman noticed unsteady gait at 33 years of age. Afterwards, dysarthria and writing difficulty were appeared. Her family history revealed that her grandfather and her mother had a clinical history of unsteadiness and mental changes. At 1 year after clinical onset, neurological examination showed cerebellar ataxia and leg hyperreflexia. At 4 years after onset, she suddenly developed insomnia and nocturnal howling. Her mental status disclosed marked disorientation, anxiety and irritability. Muscle stretch reflexes were increased in four extremities with Babinski's signs. Remarkable dysarthria and cerebellar ataxia were presented. Brain diffusion weighted imaging showed extensive hyperintensity signal areas in the cerebral cortex. A point mutation of the prion protein gene (PRNP) at codon 102 resulting in the substitution of proline by leucine (P102L) was identified. PRNP polymorphism exhibited homozygous methionine at codon 129 and homozygous glutamate at codon 219 . She had verbal perseveration, somnolence and myoclonus of lower limbs, leading to akinetic mutism at 4 months after neuropsychiatric events. Phenotypic hallmark of our patient indicates leg hyperreflexia from an early disease course. This neurological sign differs from the previously reported clinical expression of Japanese and foreign patients with GSS (P102L). Thus, physicians should pay more attention to phenotypic heterogeneity in this prion disease.
\end{abstract}

Key words: Gerstmann-Sträussler-Scheinker Syndrome, PRNP, P102L, phenotypic heterogeneity, leg hyperreflexia

(Inter Med 49: 339-342, 2010)

(DOI: 10.2169/internalmedicine.49.2864)

\section{Introduction}

The human prion protein gene (PRNP) governs most of the clinical and pathological features of prion diseases and plays an important role in the determination of host susceptibility (1). Gerstmann-Sträussler-Scheinker Syndrome (GSS) is one of the fatal inherited prion diseases. Seven point mutations of PRNP codon 102,105, 117, 145, 198, 202 and 217 are detected in GSS patients. A point mutation at PRNP codon 102 resulting in the substitution of proline to leucine (P102L) is the most common in Japanese patients with GSS
(GSS102) (2). In general, GSS102 is characterized by midlife onset of cerebellar ataxia followed by slowly progressive dementia. Linked studies of phenotypes and PRNP genotypes are reported in different ethnic families with GSS 102 (3-9). Clinicogenetic associations are found in British families with GSS102 (3). Phenotypic features of GSS102 highlight leg hyporeflexia as an early neurological sign $(4,5,9)$. Although comparative analyses between phenotypes and PRNP polymorphism have been performed in a few Japanese families with GSS102 $(4,7,8)$, detailed clinical heterogeneity remains unclarified in Japanese patients. Here, we report a distinct phenotype of leg hyperreflexia in 


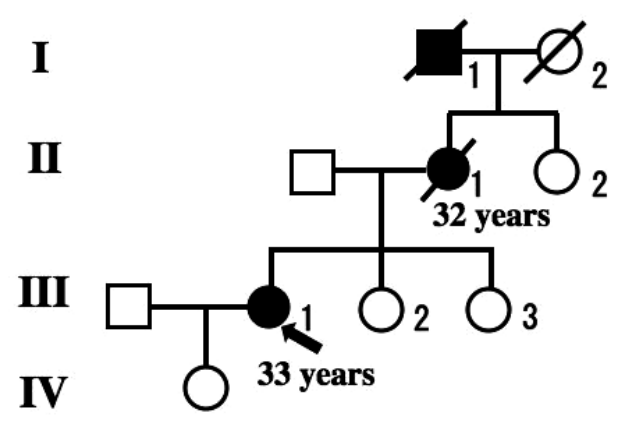

Figure 1. Pedigree of Japanese family with GSS102. Members affected by GSS are shown by solid symbols. Squares and circles represent man and woman members, respectively. Arrow indicates the proband. The age listed below symbols indicates the age at onset.

a Japanese family with GSS102.

\section{Case Report}

A 38-year-old healthy woman (III-1, the proband) developed unsteady gait at 33 years of the age. She had dysarthria and writing disturbance. Family history showed her grandfather (I-1) and her mother (II-1) had unsteadiness and mental changes (Fig. 1). Her mother noticed unbalanced walking at 32 years of age. The maternal medical record exhibited cerebellar ataxia and increased muscle stretch reflexes in the knees and the ankles. Her mother (II-1) showed personality changes at 36 years of age and died of pneumonia at 37 years old. In her grandfather (I-1), unsteady gait started from midlife age and mental changes occurred later. Regarding the present patient (III-1) at age 34 years, the initial neurological examination revealed dysarthria, hyperreflexia in the lower extremities and cerebellar ataxia in the body and four limbs. The first brain magnetic resonance imaging (MRI) showed mild degree of atrophy in the vermis (Fig. 2). No abnormalities existed in the cerebrum and the basal ganglia. Informed consent of gene study was obtained from the patient and her family. Gene analysis of spinocerebellar ataxia (SCA) types 1, 2, 3 and 6 suggested negative results. Cerebellar ataxia progressed slowly, but her mental state and intelligence were normal at 37 years of age. At 5 years after clinical onset, she suddenly developed intractable insomnia and nocturnal howling, and was admitted to our department. Physical examination showed body mass index of $17.5 \mathrm{~kg} / \mathrm{m}^{2}$. Disorientation, anxiety and irritable state were obvious. Muscle stretch reflexes were increased in four extremities with Babinski signs. Marked cerebellar ataxia and dysarthria were not altered as compared to pre-onset of mental symptoms. Remaining neurological examination was normal, including pseudobulbar signs. Routine laboratory tests were normal. On cerebrospinal fluid study, cell count $\left(1 / \mathrm{mm}^{3}\right)$ and protein $(27 \mathrm{mg} / \mathrm{dL})$ were normal. Neuronspecific enolase levels were increased to $28.7 \mathrm{ng} / \mathrm{mL}$ (normal <12.0). Total tau was elevated at more than $1,300 \mathrm{pg} /$

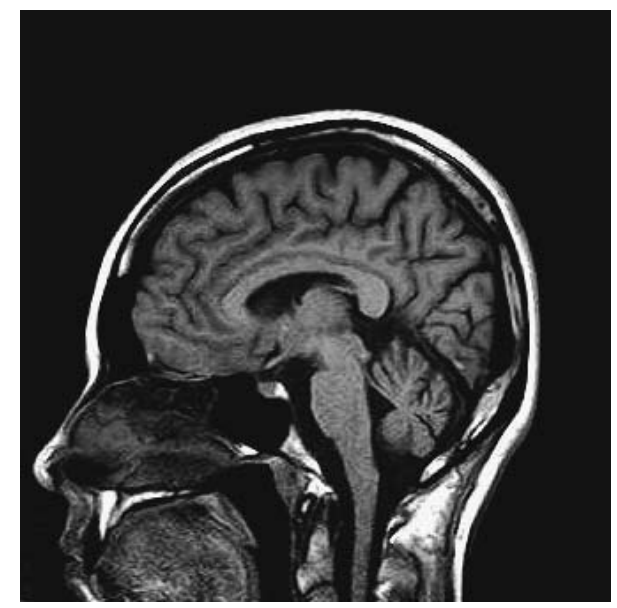

Figure 2. Mild degree of vermian atrophy is shown on T1weighted imaging before onset of mental symptoms.

$\mathrm{mL}$ and 14-3-3 protein was positive. Electromyogram was normal. Electroencephalogram showed diffuse delta and theta waves without periodic synchronous discharges. Brain MRI was performed at 1 week after onset of neuropsychiatric changes. Diffusion weighted and fluid-attenuated inversion imaging showed remarkable hyperintensity signal areas in the cerebral cortex, predominantly in the fronto-parietal regions (Fig. 3). We obtained informed consent of PRNP analysis from her family. Genomic DNA was extracted from peripheral lymphocytes in our patient (III-1, the proband). P102L mutation, homozygous methionine at PRNP codon 129 and homozygous glutamate at PRNP codon 219 were identified in our patient. At two months after admission, she had verbal perseveration, somnolence, myoclonus of lower limbs and akinetic seizure. Two months later, she had akinetic mutism and was transferred to another hospital.

\section{Discussion}

We reported a Japanese family with GSS102 in whom leg hyperreflexia was highlighted as a neurological sign in an early stage before mental events. Age at onset was 33 years in the proband (III-1) and 32 years in her mother (II-1). Her grandfather (I-1) had midlife onset of unsteadiness. Neurological characteristics of the two patients (II-1 and III-1) suggested leg hyperreflexia and slowly progressive cerebellar ataxia followed by rapidly progression of neuropsychiatric changes. In particular, the proband (III-1) showed leg hyperreflexia at an early stage. Muscle stretch reflexes were increased in four extremities after onset of neuropsychiatric changes. In addition to $\mathrm{P} 102 \mathrm{~L}$ mutation, PRNP polymorphism confirmed methionine/methionine at codon 129 and glutamate/glutamate at codon 219 .

On a recent Japanese survey of 128 patients with inherited prion disease, P102L mutation accounts for $19.6 \%$ (2). The most typical phenotype of GSS102 reveals adult-onset (50s years) of cerebellar ataxia and subsequently slowly progressive dementia. GSS102 is reported widely in patients of 


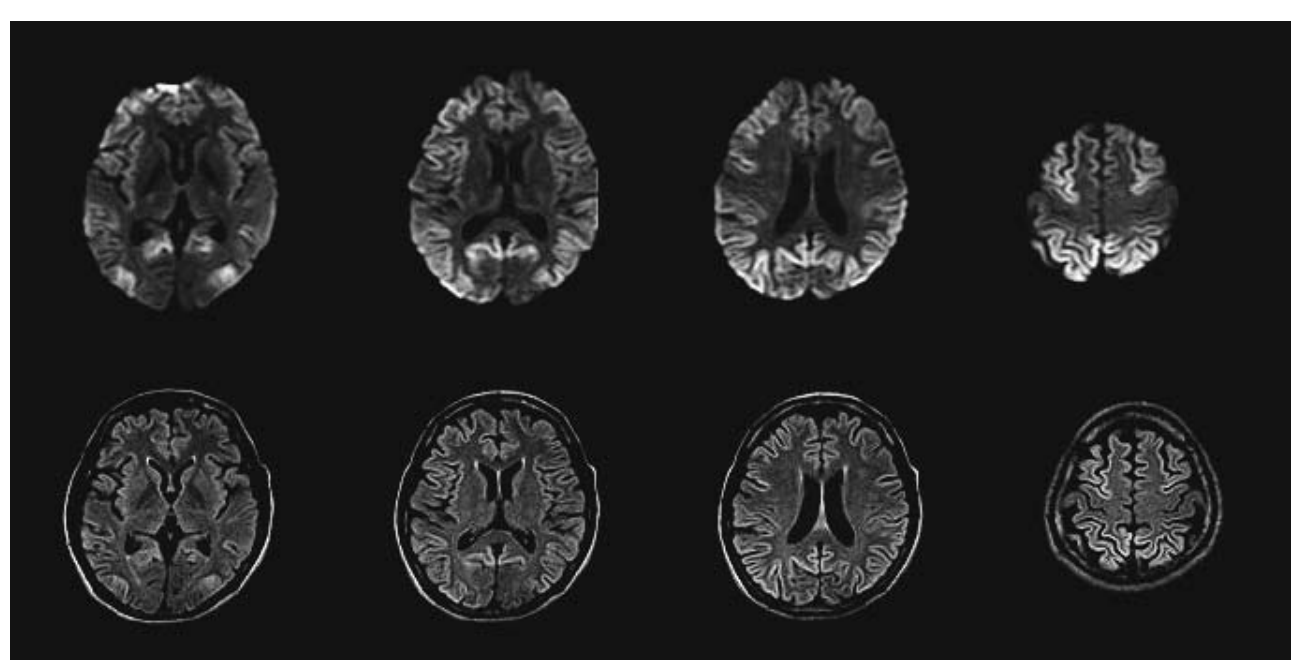

Figure 3. After sudden onset of neuropsychiatric changes, marked hyperintensity signal areas are shown predominantly in the frontoparietal cortex on diffusion weighted (upper) and fluid-attenuated inversion imaging (lower).

Austrian, England, British-Canadian, French, German, Italian, Israeli, American, Chinese and Japanese origin. The relationship between the phenotypic heterogeneity and DNA allele of PRNP codon 129 or 219 has been analyzed in several countries in families with P102L mutation (3-9). The largest investigation was carried out in 84 patients (30 men and 54 women) of 15 international families with GSS102 originating from central England (3). The neurological profile disclosed that leg areflexia accounted for $79 \%$ and no patient had hyperreflexia (3). Clinicogenotypic data support that polymorphism at PRNP codon 129 influences disease onset of GSS102. As compared to heterozygotes of methionine/valine, age at onset is younger at a mean of 7 years in homozygotes of methionine (3). Furthermore, this study suggests first that apolipoprotein E4 genotypes reflect age of onset in 22 patients. Apolipoprotein E4 carriers display later onset of 10 years in comparison with non-E4 carriers (3). In eight patients of a large Italian family with GSS102, clinical phenotypes were not associated with genotypes at PRNP codon 129 and 219 (5). Affected members of this Italian family revealed leg areflexia in the lower extremities (5). A large Chinese family with GSS102 was reported recently (6). Three surviving patients and 6 asymptomatic carriers contain homozygous methionine at PRNP codon 129 and homozygous glutamate at PRNP codon 219. Those clinicogenotypic results also provide no linkages between genotypes and clinical expressions. Loss of muscle stretch reflexes was described in two of 7 patients (6). As compared to heterogeneous phenotypes in Japanese families $(4,7,8)$, British families (3), an Italian family (5), a Chinese family (6) and an original Austrian kindred (10) with GSS102, a distinct feature of our patients (II-1 and III-1) was leg hyperreflexia at early stage before onset of mental changes. A previous large Japanese study showed the clinicoradiological findings in eleven patients of 9 unrelated families with GSS 102 (4). All patients had areflexia, dysesthesia and ataxia in the lower extremities on initial neurological examination. This neurological triad contributes to a critical key of early diagnosis of GSS102 (4). Therefore, leg hyperreflexia has not been reported in previous Japanese or foreign families with GSS102 (3-9).

As far as polymorphism of PRNP, the frequency of homozygous methionine at codon 129 is very high in Japanese population. The present three affected members (I-1, II-1 and III-1) came from Gunma Prefecture, East Japan. Eleven patients of 9 Japanese kindreds with GSS102 were born in Kagoshima Prefecture, South Japan (4). Molecular analysis of PRNP demonstrates methionine homozygotes at codon 129 and glutamate homozygotes at codon 219 in all of the patients (4). Those patients and our patient (III-1) express the same PRNP genotype whereas neurological signs of muscle stretch reflexes were opposite between our family and the Kagoshima-born families. Prion protein deposition in the lumbar posterior horns contributes to leg hyporeflexia and dysesthesia in GSS102 (11). Variable degrees of cerebral spongiosis are generated in GSS102 patients (9). Before prion protein accumulation in the spinal posterior horns, neurological damage of the cerebral cortex and the corticospinal tract may advance in our patients (II-1 and III-1). Such neuropathological changes may cause leg hyperreflexia.

Before presentation of dementia in GSS102 patients, prominent cerebellar ataxia occasionally triggers a misdiagnosis of SCA. Seven mutations of PRNP were examined in 206 Italian patients with adult-onset cerebellar ataxia who showed negative results on genetic tests of SCA type 2, 6, 7 and Friedreich ataxia. P102L mutation was found in one patient (12). That patient was a 52-year-old woman without family history who developed gait unbalance. Neurological examination revealed cerebellar ataxia, diffuse hyperreflexia and normal cognitive function (12). These neurological aspects are similar to our two patients (II-1 and III-1). Thus, 
more attention should be paid to the differential diagnosis of GSS102 in SCA gene-negative patients who have ataxia and hyperreflexia. Finally, further genetic and neuropathological analyses for novel modifying factors on phenotypes are needed to elucidate such clinicogenotypic variability of GSS 102 patients.

\section{Acknowledgement}

We thank Prof. Tetsuyuki Kitamoto, Division of CJD Science and Technology, Department of Prion Research, Tohoku University Graduate School of Medicine, Sendai, Japan for an analysis of PRPN. We are grateful to Dr. Katsuya Sato, The First Department of Internal Medicine, Nagasaki University School of Medicine, Nagasaki, Japan for the measurement of total tau and 14-33 protein.

\section{References}

1. Pocchiari M, Poleggi A, Principe S, Graziano S, Cardone F. Genomic and post-genomic analyses of human prion diseases. Genome Med 1: 63.1-63.8, 2009.

2. Yamada M. Prion diseases in Japan: Analysis of 918 patients. Clin Neurol (Tokyo) 47: 805-808, 2007.

3. Webb TEF, Poulter M, Beck J, et al. Phenotypic heterogeneity and genetic modification of P102L inherited prion disease in an international series. Brain 131: 2632-2646, 2008.

4. Arata H, Takashima H, Hirano R, et al. Early clinical signs and imaging findings in Gerstmann-Sträussler-Scheinker syndrome (Pro102Leu). Neurology 66: 1672-1678, 2006.

5. Barbanti P, Fabbrini G, Salvatore M, et al. Polymorphism at codon 129 or codon 219 of PRNP and clinical heterogeneity in a previously unreported family with Gerstmann-Sträussler-Scheinker disease (PrP-P102L mutation). Neurology 47: 734-741, 1996.

6. Chi NF, Lee YC, Lu YC, Wu HM, Soong BW. Transmissible spongiform encephalopathies with P102L mutation of PRNP manifesting different phenotypes: Clinical, neuroimaging, and electrophysiological studies in Chinese kindred in Taiwan. J Neurol: Published online: Aug 21, 2009.

7. Tanaka Y, Minematsu K, Moriyasu H, et al. A Japanese family with a variant of Gerstmann-Sträussler-Scheinker disease. J Neurol Neurosurg Psychiatry 62: 454-457, 1997.

8. Furukawa H, Kitamoto T, Tanaka Y, Tateishi J. New variant prion protein in a Japanese family with Gerstmann-Sträussler syndrome. Brain Res Mol Brain Res 30: 385-388, 1995.

9. Young K, Jones CK, Piccardo $\mathrm{P}$, et al. Gerstmann-SträusslerScheinker disease with mutation at codon 102 and methionine at codon 129 of PRNP in previously unreported patients. Neurology 45: 1127-1134, 1995.

10. Hainfellner JA, Brantner-Inthaler S, Cervenáková L, et al. The original Gerstmann-Sträussler-Scheinker family of Austria: divergent clinicopathological phenotypes but constant PrP genotype. Brain Pathol 5: 201-211, 1995.

11. Yamada M, Tomimitsu $H$, Yokota $T$, et al. Involvement of the spinal posterior horn in Gerstmann-Sträussler-Scheinker disease (PrP P102L). Neurology 52: 260-265, 1999.

12. Cagnoli C, Brussino A, Sbaiz L, et al. A previously undiagnosed case of Gerstmann-Sträussler-Scheinker disease revealed by PRNP gene analysis in patients with adult-onset ataxia. Mov Disord 23: 1468-1471, 2008.

(C) 2010 The Japanese Society of Internal Medicine http://www.naika.or.jp/imindex.html 